\title{
LỰA CHỌN HỆ TỌA Độ ĐỂ XÁC LẬP HỆ QUY CHIẾU TRONG XÂY DỤ゙NG CÔNG TRİ̀H NGẦM
}

\author{
NGUYẼ̃N QUANG THÁNG ${ }^{(1)}$, NGUYẼ̃ HÀ(1), DIÊM CÔNG HUY(2) \\ (1)Trường Đại học Mỏ - Địa chất \\ ${ }^{(2)}$ Viện Khoa học công nghệ Xây dựng
}

\section{Tóm tắt:}

Trong bài báo đã nghiên cứu, khảo sát lý thuyết và thực nghiệm để đánh giá độ lệch tọa độ địa diện chân trời của điểm do ảnh hưởng của độ chênh cao; ảnh hưởng của độ lệch dây dọi đến tọa độ địa diện trong phạm vi 10km. Nội dung bài báo cũng khảo sát đặc điểm biến dạng của tọa độ địa diện chân trời và tọa độ UTM địa phương theo kích thước vùng xét và chênh lệch độ cao điểm, trong đó có khu vực miền núi với chênh cao lớn. Từ đó đề xuất sử dụng hệ tọa độ địa diện để xác lập hệ quy chiếu trong xây dụ̂ng đường hầm đào đối hướng trên khu vực đồng bằng và miền núi.

\section{1. Đặt vấn đề}

Việc ứng dụng hệ tọa độ địa diện để xác lập hệ quy chiếu trong xây dựng công trình đã được trình bày trong một số công trình $[1,2]$. Trong những tài liệu này đã xem xét ứng dụng hệ tọa độ địa diện đối với công trình dạng vùng: công trình công nghiệp, dân dụng... trên phạm vi xây dựng không lớn.

Trong công trình [2] đã khảo sát, đánh giá và so sánh khả năng ứng dụng hệ tọa độ địa diện và hệ tọa độ vuông góc phẳng UTM để lập hệ quy chiếu trong xây dựng công trình. Tuy nhiên còn một số vấn đề chưa được xem xét, đó là ảnh hưởng của độ chênh cao điểm xét so với điểm gốc đến tọa độ địa diện của điểm, vấn đề ảnh hưởng của độ lệch dây dọi đến tọa độ điểm.

Công trình ngầm là dạng công trình xây dựng phổ biến hiện nay với một số dạng đặc trưng như: đường tàu điện ngầm; đường hầm thủy lợi, thủy điện. Một trong những đặc điểm chủ yếu trong thi công dạng công trình này là thực hiện việc đào hầm đối hướng với yêu cầu đào thông hầm trong hạn sai cho phép. Để đảm bảo đào thông hầm, nhiệm vụ công tác trắc địa bao gồm: xây dựng lưới khống chế trắc địa trên mặt đất, chuyền tọa độ và phương vị xuống dưới hầm, thành lập lưới khống chế trong hầm và bố trí đường hầm. Thông thường đoạn đường hầm đào đối hướng có chiều dài không lớn (trừ các đường hầm vượt biển).

Theo các tài liệu về công tác trắc địa công trình ngầm, khi chiều dài đoạn đường hầm đào đối hướng khoảng $4 \mathrm{~km}$, sai số trung phương hướng ngang đào thông hầm không vượt quá $\pm 50 \mathrm{~mm}$; khi chiều dài đoạn đường hầm từ $4 \div 8 \mathrm{~km}$, sai số trung phương hướng ngang không vượt quá $\pm 75 \mathrm{~mm}$; sai số trung phương độ cao đào thông hầm trong các trường hợp nêu trên không vượt quá $\pm 25 \mathrm{~mm}$.

Để xác lập hệ quy chiếu trong xây dựng đường hầm đào đối hướng chúng ta sẽ xem xét hai hệ tọa độ thường được ứng dụng trong trắc địa công trình: hệ tọa độ địa diện và hệ tọa độ vuông góc UTM; khảo sát đặc điểm biến dạng của tọa độ địa diện chân trời và tọa độ UTM địa phương theo kích thước 
vùng xét, chênh lệch độ cao điểm và độ lệch dây dọi.

\section{Hệ tọa độ địa diện và hệ tọa độ vuông góc UTM}

2.1. Hệ tọa độ địa diện và một số công thức cơ bản

Khái niệm hệ tọa độ địa diện chân trời được nêu chi tiết trong [1]. Hệ tọa độ này có gốc tọa độ tại điểm $\mathrm{O}$ được lựa chọn phù hợp (thường trùng với điểm trọng tâm của khu vực xây dựng công trình) có tọa độ trắc

$$
\left[\begin{array}{l}
x \\
y \\
z
\end{array}\right]=\left[\begin{array}{cc}
-\sin B_{0} \cdot \cos L_{0} & -\sin B_{0} \cdot \sin L_{0} \\
-\sin L_{0} & \cos L_{0} \\
\cos B_{0} \cdot \cos L_{0} & \cos B_{0} \cdot \sin L_{0}
\end{array}\right.
$$

trong đó: $\mathrm{B}_{0}, \mathrm{~L}_{0}, \mathrm{H}_{0}$ - tọa độ trắc địa của điểm gốc;

$\mathrm{N}_{0}$ - bán kính cong vòng thẳng đứng thứ nhất đi qua điểm gốc của hệ tọa độ địa diện; e - tâm sai thứ nhất của Elipxoit WGS-84;

( $x, y, z),(X, Y, Z)$ - tọa độ địa diện chân trời và tọa độ vuông góc không gian địa tâm của điểm xét.

Tọa độ vuông góc không gian địa tâm và tọa độ trắc địa có quan hệ với nhau theo công thức:

$$
\begin{aligned}
& X=(N+H) \cdot \cos B \cdot \cos L \\
& Y=(N+H) \cdot \cos B \cdot \sin L \\
& Z=\left[N\left(1-e^{2}\right)+H\right] \cdot \sin B
\end{aligned}
$$

với $\mathrm{N}$ được tính theo công thức:

$$
N=\frac{a}{\sqrt{1-e^{2} \cdot \sin ^{2} B}}
$$

Từ công thức (1), khi biết vec tơ tọa độ địa diện chân trời có thể dễ dàng tính được tọa độ vuông góc không gian địa tâm và tọa độ trắc địa. Vec tơ gia số tọa độ vuông góc không gian địa tâm $\left[\left(X-X_{0}\right)\left(Y-Y_{0}\right)(Z-\right.$ $\left.\left.Z_{0}\right)\right]^{\top}$ nhận được bằng cách lấy tích ma trận địa $\left(\mathrm{B}_{0}, \mathrm{~L}_{0}, \mathrm{H}_{0}\right)$; trục $\mathrm{Oz}$ có phương trùng với phương pháp tuyến của elipxoit quy chiếu (elipxoit WGS-84), chiều hướng từ tâm ra bên ngoài; trục $O x$ là giao tuyến giữa mặt phẳng kinh tuyến đi qua điểm $\mathrm{O}$ và mặt phẳng vuông góc với pháp tuyến tại $\mathrm{O}$ (mặt phẳng gốc), hướng về phía bắc; trục $\mathrm{Oy}$ vuông góc với trục $\mathrm{Ox}$ và $\mathrm{Oz}$, hướng về phía đông.

Tọa độ địa diện chân trời được tính theo tọa độ vuông góc không gian và tọa độ trắc địa dựa vào công thức sau [1]:

$$
\left.\begin{array}{c}
\cos B_{0} \\
0 \\
\sin B_{0}
\end{array}\right] \cdot\left[\begin{array}{l}
X-\left(N_{0}+H_{0}\right) \cdot \cos B_{0} \cdot \cos L_{0} \\
Y-\left(N_{0}+H_{0}\right) \cdot \cos B_{0} \cdot \sin L_{0} \\
Z-\left[N_{0}\left(1-e^{2}\right)+H_{0}\right] \cdot \sin B_{0}
\end{array}\right]
$$

xoay nghịch đảo và vec tơ tọa độ địa diện chân trời; từ đó tính được vec tơ tọa độ vuông góc không gian địa tâm và vec tơ tọa độ trắc địa của điểm. Có nghĩa là việc tính chuyển giữa tọa độ địa diện chân trời, tọa độ vuông góc không gian địa tâm và tọa độ trắc địa có thể thực hiện một cách dễ dàng với các công thức dạng đơn giản.

Tiếp theo chúng ta sẽ xem xét ảnh hưởng của độ chênh cao điểm xét so với điểm gốc của hệ tọa độ địa diện chân trời tới tọa độ địa diện chân trời của điểm này.

Ký hiệu: $\delta \mathrm{H}=\mathrm{H}-\mathrm{H}_{0}$, trong đó $\mathrm{H}$ - độ cao trắc địa của điểm xét; $\mathrm{H}_{0}$ - độ cao trắc địa của điểm gốc. Từ công thức (2) sau khi biến đổi sẽ có nhóm công thức tính độ chênh tọa độ địa tâm theo $\delta \mathrm{H}$ (đối với các điểm nằm trên cùng một pháp tuyến với elipxoit):

$$
\begin{aligned}
& \delta X=\cos B \cdot \cos L \cdot \delta H \\
& \delta Y=\cos B \cdot \sin L \cdot \delta H \\
& \delta Z=\sin B \cdot \delta H
\end{aligned}
$$

trong đó: $\delta X=X^{\prime}-X ; \delta Y=Y^{\prime}-Y ; \delta Z=$ $Z^{\prime}-Z$, với $(X, Y, Z),\left(X^{\prime}, Y^{\prime}, Z^{\prime}\right)$ lần lượt là tọa độ vuông góc địa tâm của điểm có độ cao trắc địa là $\mathrm{H}_{0}$ và của điểm xét có độ cao trắc địa là $\mathrm{H}$ (hai điểm này nằm trên cùng một 
đường pháp tuyến).

Từ công thức (1) sau khi biến đổi sẽ có nhóm công thức tính độ lệch của tọa độ địa diện chân trời theo $\delta \mathrm{H}$ như sau:

$$
\begin{aligned}
& \delta x=-\sin B_{0} \cos \mathrm{L}_{0} \delta X-\sin B_{0} \sin L_{0} \delta Y+\cos B_{0} \delta Z \\
& \delta y=-\sin L_{0} \delta X+\cos \mathrm{L}_{0} \delta Y+0 . \delta Z \\
& \delta z=\cos B_{0} \cos L_{0} \delta X+\cos B_{0} \sin L_{0} \delta Y+\sin B_{0} \delta Z
\end{aligned}
$$

trong đó $\delta X, \delta Y, \delta Z$ được tính theo công thức (4). Công thức (5) cũng được sử dụng để tính độ lệch gia số tọa độ địa diện chân trời theo gia số tọa độ địa tâm.

Trong trắc địa công trình thường sử dụng hệ tọa độ địa diện quy ước có các trục tọa độ song song với trục chính của công trình. Gốc tọa độ địa diện quy ước có thể chọn tại góc tây nam của khu vực xây dựng, nhưng tốt nhất là chọn trùng với gốc của hệ tọa độ địa diện chân trời. Đây là đặc điểm nổi bật và cũng là một ưu điểm của hệ tọa độ địa diện quy ước.

Để tính chuyển tọa độ địa diện chân trời của các điểm sang tọa độ địa diện quy ước sử dụng thuật toán biến đổi đồng dạng (thường là thuật toán Helmert) dựa vào ít nhất hai điểm có tọa độ trong cả hai hệ [1, 2].

\subsection{Hệ tọa độ vuông góc UTM}

Ở nước ta hiện nay để xác lập hệ tọa độ nhà nước và địa phương sử dụng phép chiếu UTM. Trong trắc địa công trình, để hạn chế biến dạng do phép chiếu sử dụng hệ tọa độ vuông góc UTM địa phương với múi chiếu $6^{\circ}$ hoăc $3^{\circ}$, kinh tuyến trục nằm cách khu vực xây dựng công trình tương ứng là $180 \mathrm{~km}$ và $90 \mathrm{~km}$. Công thức tính chuyển giữa tọa độ vuông góc UTM địa phương và các tọa độ khác được trình bày cụ thể trong [2].

\section{3. Đặc điểm biến dạng khi sử dụng hệ tọa độ địa diện và hệ tọa độ vuông góc UTM trên vùng đồng bằng}

3.1. Đặc điểm biến dạng khi sử dụng hệ tọa độ địa diện trên vùng đồng bằng
Như đã nói ở trên, mặt phẳng xOy của hệ tọa độ địa diện chân trời vuông góc với pháp tuyến của elipxoit quy chiếu, còn việc đo đạc bố trí công trình được thực hiện trên mặt thủy chuẩn (mặt vuông góc với phương dây dọi) đi qua điểm gốc tọa độ. Góc giữa hai mặt này chính là độ lệch dây dọi. Chúng ta sẽ xem xét độ lệch dây dọi ảnh hưởng đến tọa độ địa diện chân trời của các điểm như thế nào.

Ký hiệu: v - độ lệch dây dọi; S - khoảng cách giữa điểm xét và điểm gốc của hệ tọa

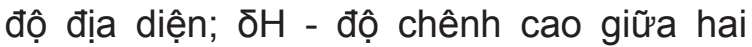
điểm này, $\delta \mathrm{H}$ có thể tính theo công thức:

$$
\delta H=\frac{v}{\rho} \cdot S
$$

Ở vùng đồng bằng có thể lấy v = 10", với $\rho=206264,8062 " ; S=5 \mathrm{~km}$ thì $\delta \mathrm{H}=$ $242.4 \mathrm{~mm}$; với $\mathrm{S}=10 \mathrm{~km}, \delta \mathrm{H}=484.8 \mathrm{~mm}$.

Để khảo sát biến dạng, tiến hành tính toán độ lệch tọa độ địa diện chân trời theo công thức (5) cho các khu vực có bán kính lần lượt là $R=5 \mathrm{~km}$ và $R=10 \mathrm{~km}$ trên địa bàn Hà Nội. Có 8 điểm xét bao gồm 2 điểm trên hướng ngang, 2 điểm trên hướng dọc và 4 điểm nằm trên các hướng tạo với trục tọa độ góc $45^{\circ}$, các hướng này đều đi qua điểm gốc. Độ chênh cao $\delta \mathrm{H}$ đưa vào tính toán là $242.4 \mathrm{~mm}$ và $484.8 \mathrm{~mm}$ ứng với hai bán kính nêu trên.

Ngoài ra để khảo sát biến dạng khi sử dụng hệ tọa độ địa diện còn tiến hành tính toán độ lệch tọa độ địa diện chân trời theo độ chênh cao $\delta \mathrm{H}=31.85 \mathrm{~m}$ cho các khu vực có bán kính là $R=5 \mathrm{~km}$ và $R=10 \mathrm{~km}$ cũng ở địa bàn trên.

Kết quả tính toán độ lệch toán độ lệch tọa độ địa diện chân trời trong các trường hợp nêu trên được trình bày ở bảng 1 . Ở đây cần lưu ý rằng $\delta x, \delta y, \delta z$ và $\delta H$ có cùng đơn vị (mm hoặc $\mathrm{m})$. Giá trị 1/T được tính theo công thức:

$$
\frac{1}{T}=1:\left(\frac{R}{\sqrt{(\delta x)^{2}+(\delta y)^{2}}}\right)
$$

Từ kết quả tính toán độ lệch $\delta x, \delta y, \delta z$ 
theo $\delta \mathrm{H}$ nêu ở bảng 1 , có thể rút ra một số nhận xét sau đây:

1) Độ lệch tọa độ địa diện chân trời do ảnh hưởng của độ lệch dây dọi ở vùng đồng bằng có giá trị rất nhỏ: với $R=10 \mathrm{~km}$ thì $(\delta \mathrm{x}$, бу) $\max =0.8 \mathrm{~mm},(1 / \mathrm{T}) \max =$ 1/13.131.000. Điều đó có nghĩa là hoàn toàn có thể sử dụng tọa độ địa diện chân trời trên mặt phẳng vuông góc với pháp tuyến của elipxoit trong đo đạc bố trí công trình trên mặt thủy chuẩn.

2) Độ lệch tọa độ tổng hợp về mặt bằng từ tâm đến điểm xét trong các trường hợp tính là bằng nhau, nghĩa là độ lệch này đồng đều theo các hướng tính từ điểm gốc. Càng xa điểm gốc giá trị độ lệch càng tăng lên.

3) Khi các điểm xét có cùng vĩ độ với điểm gốc (nằm trên hướng trục $\mathrm{Oy}$ ) thì độ lệch tọa độ địa diện tối đa đạt được trên hướng trục Oy, còn độ lệch tọa độ địa diện trên hướng trục $O x$ bằng 0 ; còn khi các điểm xét có cùng tung độ với điểm gốc (nằm trên hướng trục $\mathrm{Ox}$ ) thì độ lệch tọa độ địa diện tối đa đạt được trên hướng trục $O x$, còn độ lệch tọa độ địa diện trên hướng trục Oy bằng 0 .

4) Khi độ chênh cao $\delta \mathrm{H} \leq 31.85 \mathrm{~m}$, giá trị $(1 / T) \leq 1 / 200.000$, đáp ứng được hầu hết yêu cầu về độ chính xác của công tác trắc địa công trình. (Xem bảng 1)

3.2. Đặc điểm biến dạng khi sử dụng hệ tọa độ vuông góc UTM trên vùng đồng bằng

Từ các khảo sát trong [2], có thể nêu một số nhận xét về đặc điểm biến dạng đối với hệ tọa độ vuông góc UTM trên vùng đồng bằng như sau:

- Biến dạng về chiều dài do phép chiếu không đồng đều theo các hướng, có giá trị như nhau khi tung độ trung bình $\mathrm{y}_{\mathrm{m}}$ bằng nhau.

- Khi độ cao mặt chiếu $\delta H \leq 31.85 \mathrm{~m}$, giá trị biến dạng dài tương đối $(1 / T) \leq$ $1 / 200.000$.

\section{4. Đặc điểm biến dạng khi sử dụng hệ tọa độ địa diện và hệ tọa độ vuông góc UTM ở vùng núi}

4.1. Đặc điểm biến dạng khi sử dụng hệ tọa độ địa diện ở vùng núi

Để khảo sát biến dạng, tiến hành tính toán độ lệch tọa độ địa diện chân trời theo công thức (5) cho hai phương án có bán kính lần lượt là $R=5 \mathrm{~km}$ và $R=2.5 \mathrm{~km}$ trên địa bàn tỉnh Gia Lai.

Phương án 1: bán kính vùng tính là $R=$ $5 \mathrm{~km}$, tọa độ trắc địa của điểm gốc: $\mathrm{B}_{0}=$ 14'13'50"; $L_{0}=107^{\circ} 49^{\prime} 50^{\prime \prime} ; H_{0}=500 m ; \delta H$ được chọn bằng $100 \mathrm{~m}$ và $200 \mathrm{~m}$. Ngoài ra để tính ảnh hưởng của độ lệch dây dọi đến tọa độ địa diện, chọn $\delta \mathrm{H}=484.8 \mathrm{~mm}$ ứng với $v=20$ " cho vùng núi.

Phương án 2: bán kính vùng tính là $R=$ $2.5 \mathrm{~km}$, tọa độ trắc địa của điểm gốc: $\mathrm{B}_{0}=$ 14.13'50"; $L_{0}=107^{\circ} 51^{\prime} 11^{\prime \prime} ; H_{0}=550 \mathrm{~m}$ và $600 \mathrm{~m}$; $\delta \mathrm{H}$ được chọn bằng $50 \mathrm{~m}$ và $100 \mathrm{~m}$. Có nghĩa là về mặt bằng điểm gốc nằm ở điểm giữa bán kính theo phương vĩ tuyến về phía phải của phương án 1 ; còn về độ cao thì mặt chiếu là mặt trung bình giữa độ cao hai đầu mút của bán kính này.

Việc tính toán độ lệch tọa độ địa diện chân trời được thực hiện cũng giống như đối với vùng đồng bằng, nghĩa là tính toán cho 8 điểm, bao gồm 2 điểm trên hướng ngang, 2 điểm trên hướng dọc và 4 điểm nằm trên các hướng tạo với trục tọa độ góc $45^{\circ}$, các hướng này đều đi qua điểm gốc.

Kết quả tính toán độ lệch tọa độ địa diện chân trời theo $\delta H$ đối với hai phương án nêu trên được trình bày trong bảng 2 .

Đối với phương án 1 , độ lệch tọa độ địa diện chân trời do ảnh hưởng của độ lệch dây dọi $(\delta \mathrm{H}=484.8 \mathrm{~mm})$ có giá trị $(\delta \mathrm{x}, \delta \mathrm{y})$ 
$\max =0.4 \mathrm{~mm},(1 / \mathrm{T}) \max =1 / 13.131 .000$.

Kết quả này không được đưa vào trong Bảng 2 do hạn chế về số trang của bài báo.

\section{(Xem bảng 2)}

Từ kết quả tính toán độ lệch $\delta x, \delta y, \delta z$ theo $\delta \mathrm{H}$ nêu ở bảng 2 , có thể rút ra một số nhận xét, trong đó có 3 nhận xét trùng với 3 nhận xét đầu rút ra từ kết quả tính toán ở bảng 1 (mục 3.1). Ngoài ra còn có một số nhận xét sau:

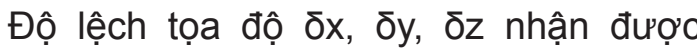
trong phương án 2 đã giảm đi 4 lần so với phương án 1: ở phương án 1: với $\delta \mathrm{H}=$ $100 \mathrm{~m},(\delta \mathrm{x}, \delta \mathrm{y}) \max =\mathbf{0 . 0 7 9 m}$; ở phương án 2: với $\delta \mathrm{H}=50 \mathrm{~m},(\delta \mathrm{x}, \delta \mathrm{y}) \max =0.020 \mathrm{~m}$; ở

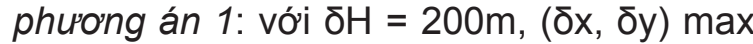
$=0.157 \mathrm{~m}$; ở phương án 2: với $\delta \mathrm{H}=100 \mathrm{~m}$, $(\delta x, \delta y) \max =0.039 m$. Có nghĩa là việc chọn điểm gốc và mặt chiếu phù hợp đã làm giảm đáng kể độ lệch tọa độ địa diện do chênh lệch độ cao $\delta \mathrm{H}$.

4.2. Đặc điểm biến dạng khi sử dụng hệ tọa độ vuông góc UTM ở vùng núi

Từ [2] có thể rút ra được công thức tính độ lệch chiều dài theo độ chênh cao như sau:

$$
\Delta S_{h}=\frac{\Delta H}{R_{m}+H_{1}} S
$$

trong đó: $\mathrm{R}_{\mathrm{m}}$ - bán kính cong trung bình của Trái đất; $\mathrm{H}_{1}$ - độ cao của điểm đầu đoạn xét. Lấy các giá trị: $\mathrm{S}=5 \mathrm{~km} ; \mathrm{R}_{\mathrm{m}}=6371 \mathrm{~km}$; $\mathrm{H}_{1}=500 \mathrm{~m}$; với $\Delta \mathrm{H}=100 \mathrm{~m}$ tính được $\Delta \mathrm{S}_{\mathrm{h}}=$ $0.079 \mathrm{~m}$, với $\Delta \mathrm{H}=200 \mathrm{~m}$ thì $\Delta \mathrm{S}_{\mathrm{h}}=0.157 \mathrm{~m}$, tức là tương đương với các giá trị nhận được ở phương án 1 trong bảng 2.

\section{Lựa chọn hệ tọa độ để xác lập hệ}

Bảng 1: Độ lệch tọa độ địa diện chân trời theo độ chênh cao $\delta H$ ở vùng đồng bằng

\begin{tabular}{|c|c|c|c|c|c|c|c|c|c|c|c|c|c|}
\hline \multicolumn{14}{|c|}{$B_{0}=21^{\circ} 01^{\prime} 40^{\prime \prime} ; L_{0}=105^{\circ} 51^{\prime} 10^{\prime \prime} ; H_{0}=0 \mathrm{~m} ; R=5 \mathrm{~km}$} \\
\hline \multicolumn{3}{|c|}{ B } & \multicolumn{3}{|c|}{ L } & \multicolumn{4}{|c|}{$\delta \mathrm{H}=242.4 \mathrm{~mm}$} & \multicolumn{4}{|c|}{$\delta \mathrm{H}=31.85 \mathrm{~m}$} \\
\hline 0 & " & " & 0 & & " & $\delta x$ & $\overline{\delta y}$ & $\delta z$ & $1 / T$ & $\delta x$ & бy & $\delta z$ & $1 / \mathrm{T}$ \\
\hline 21 & 1 & 40 & 105 & 53 & 52 & 0.0 & 0.2 & 242.4 & $1 / 28136000$ & 0.0000 & 0.0233 & 31.8500 & $1 / 214000$ \\
\hline 21 & 1 & 40 & 105 & 48 & 28 & 0.0 & -0.2 & 242.4 & $1 / 28136000$ & 0.0000 & -0.0233 & 31.8500 & $1 / 214141$ \\
\hline 21 & 4 & 22 & 105 & 51 & 10 & 0.2 & 0.0 & 242.4 & $1 / 26262000$ & 0.0250 & 0.0000 & 31.8500 & $1 / 200000$ \\
\hline 20 & 58 & 58 & 105 & 51 & 10 & -0.2 & 0.0 & 242.4 & $1 / 26262000$ & -0.0250 & 0.0000 & 31.8500 & $1 / 200000$ \\
\hline 21 & 3 & 35 & 105 & 53 & 5 & 0.1 & 0.1 & 242.4 & $1 / 27046000$ & 0.0178 & 0.0166 & 31.8500 & $1 / 206000$ \\
\hline 21 & 3 & 35 & 105 & $\overline{49}$ & 15 & 0.1 & -0.1 & 242.4 & $1 / 27046000$ & 0.0178 & -0.0166 & 31.8500 & $1 / 206000$ \\
\hline 20 & 59 & 45 & 105 & 49 & 15 & -0.1 & -0.1 & 242.4 & $1 / 27043000$ & -0.0178 & -0.0166 & 31.8500 & $1 / 206000$ \\
\hline 20 & 59 & 45 & 105 & 53 & 5 & -0.1 & 0.1 & 242.4 & $1 / 27043000$ & -0.0178 & 0.0166 & 31.8500 & $1 / 206000$ \\
\hline \multicolumn{14}{|c|}{$B_{0}=21^{\prime} 01^{\prime} 40^{\prime \prime} ; L_{0}=105^{0} 51^{\prime} 10^{\prime \prime} ; H_{0}=0 \mathrm{~m} ; R=10 \mathrm{~km}$} \\
\hline \multicolumn{3}{|c|}{$\mathrm{B}$} & \multicolumn{3}{|c|}{$\mathrm{L}$} & \multicolumn{4}{|c|}{$\delta \mathrm{H}=484.8 \mathrm{~mm}$} & \multicolumn{4}{|c|}{$\delta \mathrm{H}=31.85 \mathrm{~m}$} \\
\hline 0 & ‘ & " & 0 & & " & $\delta x$ & $\overline{\delta y}$ & $\delta z$ & $1 / \mathrm{T}$ & $\delta x$ & бy & $\delta z$ & $1 / \mathrm{T}$ \\
\hline 21 & 1 & 40 & 105 & $\overline{56}$ & 34 & $\overline{0.0}$ & 0.7 & 484.8 & $1 / 14068000$ & 0.0000 & 0.0467 & 31.8500 & $1 / 214000$ \\
\hline 21 & 1 & 40 & 105 & $\overline{45}$ & 46 & 0.0 & -0.7 & 484.8 & $1 / 14068000$ & 0.0000 & -0.0467 & 31.8500 & $1 / 214000$ \\
\hline 21 & 7 & 4 & 105 & 51 & 10 & 0.8 & 0.0 & 484.8 & $1 / 13131000$ & 0.0500 & 0.0000 & 31.8500 & $1 / 200000$ \\
\hline 20 & 56 & 16 & 105 & 51 & 10 & -0.8 & 0.0 & 484.8 & $1 / 13131000$ & -0.0500 & 0.0000 & 31.8500 & $1 / 200000$ \\
\hline 21 & 5 & 29 & 105 & 54 & 59 & 0.5 & 0.5 & 484.8 & $1 / 13583000$ & 0.0354 & 0.0330 & 31.8500 & $1 / 207000$ \\
\hline 21 & 5 & 29 & 105 & 47 & 21 & 0.5 & -0.5 & 484.8 & $1 / 13583000$ & 0.0354 & -0.0330 & 31.8500 & $1 / 207000$ \\
\hline 20 & 57 & 51 & 105 & 47 & 21 & -0.5 & -0.5 & 484.8 & $1 / 13580000$ & -0.0354 & -0.0330 & 31.8500 & $1 / 207000$ \\
\hline 20 & 57 & 51 & 105 & 54 & 59 & -0.5 & 0.5 & 484.8 & $1 / 13580000$ & -0.0354 & 0.0330 & 31.8500 & $1 / 207000$ \\
\hline
\end{tabular}


quy chiếu trong xây dựng công trình ngầm

5.1. Ứng dụng hệ tọa độ địa diện để xác lập hệ quy chiếu trong xây dựng công trình ngầm

Chúng ta thấy rằng trong xây dựng công trình ngầm, các đoạn hầm đào đối hướng có chiều dài không lớn lắm (thông thường s $5 \mathrm{~km})$.

Với điều kiện công nghệ hiện nay, thường ứng dụng công nghệ định vị vệ tinh (ở nước ta chủ yếu sử dụng công nghệ GPS) để thành lập lưới khống chế trên mặt đất trong xây dựng đường hầm đào đối hướng, trong đó ở mỗi cửa hầm hoặc miệng giếng đứng có 3 điểm: một điểm ở cửa hầm và hai điểm định hướng.
Từ những phân tích về lý thuyết và tính toán thực nghiệm nêu trên đối với hai hệ tọa độ, theo ý kiến của chúng tôi nên sử dụng hệ tọa độ địa diện để xác lập hệ quy chiếu trong thi công xây dựng đường hầm đào đối hướng, do đặc điểm chiều dài đoạn hầm không lớn, cũng như những ưu điểm đã trình bày của hệ tọa độ địa diện.

5.2. Một số vấn đề cần lưu ý khi ứng dụng hệ tọa độ địa diện để xác lập hệ quy chiếu trong xây dựng công trình ngầm

Khi ứng dụng hệ tọa độ địa diện để xác lập hệ quy chiếu trong thi công xây dựng đường hầm đào đối hướng trên các khu vực khác nhau cần lưu ý những vấn đề sau:

1) Trên khu vực đồng bằng:

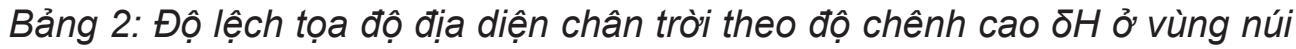

\begin{tabular}{|c|c|c|c|c|c|c|c|c|c|c|c|c|c|}
\hline \multicolumn{14}{|c|}{ Phương án 1: $B_{0}=14^{\circ} 13^{\prime} 50^{\prime \prime} ; L_{0}=107^{\circ} 49^{\prime} 50^{\prime \prime} ; H_{0}=500 \mathrm{~m} ; R=5 \mathrm{~km}$} \\
\hline \multicolumn{3}{|c|}{ B } & \multicolumn{3}{|c|}{$\bar{L}$} & \multicolumn{4}{|c|}{$\delta \mathrm{H}=100 \mathrm{~m}$} & \multicolumn{4}{|c|}{$\delta \mathrm{H}=200 \mathrm{~m}$} \\
\hline 0 & & " & 0 & & & $\delta x$ & бy & $\delta z$ & $1 / T$ & $\delta x$ & бy & $\delta z$ & $1 / T$ \\
\hline 14 & 13 & 50 & 107 & 52 & 32 & 0.000 & 0.076 & 100.000 & $1 / 65700$ & 0.000 & 0.152 & 200.000 & $1 / 32800$ \\
\hline 14 & 13 & 50 & 107 & 47 & 8 & 0.000 & -0.076 & 100.000 & $1 / 65700$ & 0.000 & -0.152 & 200.000 & $1 / 32800$ \\
\hline 14 & 16 & 32 & 107 & 49 & 50 & 0.079 & 0.000 & 100.000 & $1 / 63700$ & 0.157 & 0.000 & 200.000 & $1 / 31800$ \\
\hline 14 & 11 & 8 & 107 & 49 & 50 & -0.079 & 0.000 & 100.000 & $1 / 63700$ & -0.157 & 0.000 & 200.000 & $1 / 31800$ \\
\hline 14 & 15 & 45 & 107 & 51 & 45 & 0.056 & 0.054 & 100.000 & $1 / 64400$ & 0.112 & 0.108 & 200.000 & $1 / 32200$ \\
\hline 14 & 15 & 45 & 107 & 47 & 55 & 0.056 & -0.054 & 100.000 & $1 / 64400$ & 0.112 & -0.108 & 200.000 & $1 / 32200$ \\
\hline 14 & 11 & 55 & 107 & 47 & 55 & -0.056 & -0.054 & 100.000 & $1 / 64400$ & -0.111 & -0.108 & 200.000 & $1 / 32200$ \\
\hline 14 & 11 & 55 & 107 & 51 & 55 & -0.056 & 0.059 & 100.000 & $1 / 61700$ & -0.111 & 0.118 & 200.000 & $1 / 30900$ \\
\hline \multicolumn{14}{|c|}{ Phương án 2: $\mathrm{B}_{0}=14^{0} 13^{\prime} 50^{\prime \prime} ; \mathrm{L}_{0}=107^{\circ} 51^{\prime} 11^{\prime \prime} ; \mathrm{H}_{0}=550 \mathrm{~m}$ và $600 \mathrm{~m} ; \mathrm{R}=2.5 \mathrm{~km}$} \\
\hline \multicolumn{3}{|c|}{ B } & \multicolumn{3}{|c|}{$\mathrm{L}$} & \multicolumn{4}{|c|}{$\delta \mathrm{H}=50 \mathrm{~m}$} & \multicolumn{4}{|c|}{$\delta \mathrm{H}=100 \mathrm{~m}$} \\
\hline 0 & ، & " & 0 & & . & $\delta x$ & $\delta y$ & $\delta z$ & $1 / T$ & $\delta x$ & бy & $\delta z$ & $1 / T$ \\
\hline 14 & 13 & 50 & 107 & 52 & 32 & 0.000 & 0.019 & 50.000 & $1 / 131400$ & 0.000 & 0.038 & 100.000 & $1 / 65700$ \\
\hline 14 & 13 & 50 & 107 & 49 & 50 & 0.000 & -0.019 & 50.000 & $1 / 131400$ & 0.000 & -0.038 & 100.000 & $1 / 65700$ \\
\hline 14 & 15 & 11 & 107 & 51 & 11 & 0.020 & 0.000 & 50.000 & 1/127300 & 0.039 & 0.000 & 100.000 & $1 / 63700$ \\
\hline 14 & 12 & 29 & 107 & 51 & 11 & -0.020 & 0.000 & 50.000 & $1 / 127300$ & -0.039 & 0.000 & 100.000 & $1 / 63700$ \\
\hline 14 & 14 & 41 & 107 & 52 & 8 & 0.012 & 0.013 & 50.000 & $1 / 137200$ & 0.025 & 0.027 & 100.000 & $1 / 68600$ \\
\hline 14 & 14 & 41 & 107 & 50 & 14 & 0.012 & -0.013 & 50.000 & $1 / 137200$ & 0.025 & -0.027 & 100.000 & $1 / 68600$ \\
\hline 14 & 12 & 53 & 107 & 50 & 14 & -0.014 & -0.013 & 50.000 & $1 / 129900$ & -0.028 & -0.027 & 100.000 & $1 / 65000$ \\
\hline 14 & 12 & 53 & 107 & 52 & 8 & -0.014 & 0.013 & 50.000 & $1 / 129900$ & -0.028 & 0.027 & 100.000 & $1 / 65000$ \\
\hline
\end{tabular}


Để xác lập hệ quy chiếu, có thể chọn điểm gốc hệ tọa độ địa diện trùng với điểm cửa hầm, hoặc tại điểm giữa đoạn nối hai cửa hầm tùy thuộc vào chiều dài đoạn hầm đào đối hướng ngắn hay dài. Khi độ cao mặt chiếu nhỏ hơn hoặc bằng $\pm 31.85 \mathrm{~m}$ thì không cần lưu ý số hiệu chỉnh do độ cao mặt chiếu.

Trường hợp cần quan tâm ở đây là khi đường hầm có chiều sâu lớn (có thể đến hàng trăm mét). Khi đó nếu sử dụng mặt chiếu trùng với mặt trung bình của đường ray, cần quan tâm đến số hiệu chỉnh do độ lệch dây dọi. Ta biết rằng khi tính tọa độ địa diện sử dụng đường cơ sở là đường pháp tuyến, còn khi định hướng đường hầm tiến hành chiếu điểm cửa hầm (đã được xác định tọa độ bằng công nghệ GPS) xuống dưới hầm theo phương dây dọi.

Để xác định độ lệch dây dọi tại hai cửa hầm, có thể sử dụng phương pháp được trình bày trong [3] dựa vào độ cao trắc địa và độ cao thủy chuẩn tại ba điểm ở mỗi cửa hầm hoặc giếng đứng. Sau khi đã xác định được độ lệch dây dọi, số hiệu chỉnh vào tọa độ được tính theo công thức:

$$
V_{x}=\frac{\xi}{\rho} \cdot \Delta H ; \quad V_{y}=\frac{\eta}{\rho} . \Delta H
$$

trong đó: $\xi, \eta$ - độ lệch dây dọi thành phần trên mặt phẳng kinh tuyến và mặt phẳng thẳng đứng thứ nhất (đơn vị là giây); $\Delta \mathrm{H}$ - độ chênh cao trắc địa giữa điểm cửa hầm và mặt chiếu.

\section{2) Trên vùng núi cao:}

Khi sử dụng hệ tọa độ địa diện để xác lập hệ quy chiếu trong xây dựng đường hầm đào đối hướng trên khu vực miền núi có độ cao và chênh cao lớn, nên chọn điểm gốc hệ tọa độ địa diện tại điểm giữa của đoạn trục hầm, còn mặt chiếu được chọn có độ cao trung bình giữa hai điểm cửa hầm (đồng thời cũng đi qua điểm gốc tọa độ). Cách chọn hệ tọa độ như vậy sẽ hạn chế đến mức thấp nhất ảnh hưởng của phép chiếu và độ cao chiếu đến tọa độ địa diện của các điểm.

\section{Kết luận}

Từ kết quả nghiên cứu lý thuyết và thực nghiệm nêu trên, có thể rút ra một số kết luận như sau:

1) Nên sử dụng hệ tọa độ địa diện để xác lập hệ quy chiếu trong xây dựng đường hầm đào đối hướng do đặc điểm của công trình và ưu điểm nổi bật của hệ tọa độ này.

2) Để xác lập hệ quy chiếu trong xây dựng đường hầm đào đối hướng trên khu vực miền núi có độ cao và chênh cao lớn, nên sử dụng hệ tọa độ địa diện với điểm gốc tại điểm giữa của đoạn trục hầm, còn mặt chiếu được chọn có độ cao trung bình giữa hai điểm cửa hầm đi qua điểm gốc tọa độ. $O$

\section{Tài liệu tham khảo}

[1]. Lê Văn Hùng, 2013. Nghiên cứu bình sai kết hợp trị đo GPS và trị đo mặt đất trong hệ tọa độ vuông góc không gian địa diện chân trời áp dụng cho các mạng lưới trắc địa công trình. Luận án tiến sĩ kỹ thuật, Hà Nội 2013.

[2]. Nguyễn Quang Phúc, 2009. Nghiên cứu hoàn thiện phương pháp thành lập và xử lý số liệu lưới khống chế thi công các công trình xây dựng trong điều kiện Việt Nam. Đề tài khoa học cấp Bộ mã số B200802-52, 12/2009.

[3]. Nguyễn Quang Thắng, 2016. Luận giải ảnh hưởng của một số yếu tố đến độ chính xác chuyển trục công trình lên các sàn xây dựng trong thi công nhà siêu cao tầng. Tạp chí Khoa học kỹ thuật Mỏ - Địa chất, số 53 - 01/2016. O

(Xem tiếp trang 58) 